\title{
DISCO: A Coherent Diffeomorphic Framework for Brain Registration under Exhaustive Sulcal Constraints
}

\author{
Guillaume Auzias $^{1}$, Joan Glaunès ${ }^{2}$, Olivier Colliot ${ }^{1}$, Matthieu Perrot ${ }^{3}$, \\ Jean-François Mangin ${ }^{3}$, Alain Trouvé ${ }^{4}$, and Sylvain Baillet ${ }^{1,5}$ \\ 1 Université Pierre et Marie Curie-Paris 6, UMR 7225, UMRS 975, Centre de \\ Recherche de l'Institut Cerveau-Moelle (CRICM), Paris, France \\ 2 MAP5, Université Paris 5 - René Descartes, Paris, France \\ 3 NeuroSpin, CEA, Orsay, France \\ ${ }^{4}$ CMLA, ENS de Cachan, France \\ 5 Medical College of Wisconsin, Milwaukee, USA \\ guillaume.auzias@upmc.fr
}

\begin{abstract}
Neuroimaging at the group level requires spatial normalization of individual structural data. We propose a geometric approach that consists in matching a series of cortical surfaces through diffeomorphic registration of their sulcal imprints. The resulting 3D transforms naturally extends to the entire MRI volumes. The DIffeomorphic Sulcal-based COrtical (DISCO) registration integrates two recent technical outcomes: 1) the automatic extraction, identification and simplification of numerous sulci from T1-weighted MRI data series hereby revealing the sulcal imprint and 2) the measure-based diffeomorphic registration of those crucial anatomical landmarks. We show how the DISCO registration may be used to elaborate a sulcal template which optimizes the distribution of constraints over the entire cortical ribbon. DISCO was evaluated through a group of 20 individual brains. Quantitative and qualitative indices attest how this approach may improve both alignment of sulcal folds and overlay of gray and white matter volumes at the group level.
\end{abstract}

\section{Introduction}

Correct alignment of cortical surfaces amongst a group of subjects is crucial in neuroimaging because of the large variety of breakthrough applications. Intensitybased approaches cannot achieve this issue as the matching criteria being used is global and not optimized for finer-scale warping at the cortical level. In that respect, research in volume-based methods has evolved from the mere alignement of intesity-based elements to the integration of geometrical features of the cortical surface and even recently of cortical surface itself with hybrid surfacic and volumic approaches 912. However, as in more standard methods like 2], the resulting deformation constraints enforce the alignment of cortical valleys and crests but without any guarantee that sulci of identical anatomical denomination would be properly aligned altogether (e.g. the precentral sulcus from a 
subset of subjects might end up being matched to the central sulcus of other subjects). Specific alignment of cortical circonvolutions remains crucial as the sulcal folds shape might be correlated with the underlying functional organization 4 and requires higher level anatomical knowledge. Indeed, explicit sulcal constraints have been experimented in e.g. [14/7/13. Joshi et al. have proposed to combine a surfacic sulcal warp with a subsequent volumic matching step 8 . However, in all those approaches the similarity between sulcal landmarks relies on their parametrisation which impose strong assumptions about their topology and therefore about the segmentation. Few sulcal landmarks are then selected manually; which results in a sparse and heterogeneous distribution of anatomical landmarks guiding the transformations. Heterogeneities in the constraints favors irregularities of deformation fields and might result in alterations of the overall topology of the cortex.

In this contribution, we optimize the alignment of cerebral structures among a group of subjects through the registration of their exhaustive and sulcus-based folding patterns while constraining the dense 3D transform to be diffeomorphic - that is, smooth and invertible - through Large Deformation Diffeomorphic Metric Mapping (LDDMM) framework [5. The DISCO registration technique DIffeomophic Sulcal-based COrtical registration - consists in the following steps:

1. The automatic extraction, identification and simplification of up to 120 sulci per subject. This step yields a dense set of distributed landmarks, denoted as the individual sulcal imprint, and prevents the use of tedious and to a great extent subjective, manual tracing.

2. An LDDMM transform aiming at matching individual sulcal imprints obtained from Step 1 The similarity between corresponding sulci rely on measure based-representation which avoids the parametrisation of landmarks. We describe how this procedure can be derived from a group of subjects of arbitrary size through the definition of an adaptive sulcal template derived from the very set of subjects involved. Note that the LDDMM framework naturally yields the ability to extend the deformation to any additional object in $3 \mathrm{D}$ - such as deeper brain structures - thereby overcoming the major limitation of surface-based approaches.

The performances of DISCO are quantitatively evaluated using data from a group of 20 healthy control subjects.

\section{Extraction of the Individual Sulcal Imprint}

The extraction of the individual sulcal imprint from T1-weighted MR image volumes is initiated by the automatic segmentation and labeling of a large number of sulci using the brainVISA free software platform [1]10. Voxel labeling of the gray and white matter tissues are obtained from histogram analyses and mathematical morphological techniques applied to the biased-corrected T1weighted MR images. Elementary sulcal elements are segmented and divided into topologically-simple surfaces and organized as a graph structure. The sulci are 

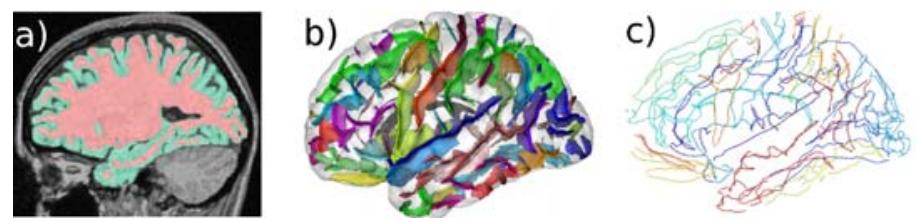

Fig. 1. Automatic extraction of the sulcal imprint: a) tissue classification; b) extraction of sulci and automatic identification results in up to 60 individual sulcal labels in each hemisphere. c) The automatic simplification of the original complex sulcal pattern yields a distributed set of sulcal edges, thereby defining the sulcal imprint of an individual brain.

then automatically labeled according to a predefined nomenclature of 60-sulcus labels per hemisphere as illustrated on Fig 1 b). Agreement between the computer and human experts reaches $86 \%$ on average [11. Though the performances of the automatic labeling procedure might reach up to $96 \%$ for well-defined folds such as the central sulcus, they tend to decline in regions where the cortical folding pattern has shows considerable inter-individual variability such as in the occipital areas.

This extraction yields structures with complex shapes made of subsets of voxels corresponding to an overly detailed description of the sulci in the context of inter-individual registration, while it is crucial to preserve the variability in topology of a given sulcus across individuals. The original complex sulcal structures are subsequently and automatically simplified as relevant sulcal landmarks using the original procedure detailed thereafter.

\subsection{From Sulci to Sulcal Imprint}

For each sulcus $s$, the initial set of voxels is first reduced to two subsets: one corresponding to the fundus of $s$, defined as the border of the sulcus reaching the deepest into the brain volumes, and one for its outer border, defined as the junction between the sulcus and the hull of the brain. These voxels are readily identified during the very sulcal extraction process as performed by brainVISA.

These two initial subsets are then independently reduced and smoothed using K-means clustering as illustrate Fig $2 \mathrm{~b}$ ). The voxels are geometrically grouped into smaller clusters which are subsequently reduced to their respective centroid. The resulting points along either sulcal border are then connected using the minimum spanning tree approach, thereby yielding two open connected graph structures, i.e. two trees (see Fig 2 ). Finally, the secondary branches of the trees are removed using a longest-path approach (Fig 2 $)$. This way, the fundus and outer borders of each sulcus $s$ are reduced to two edges denoted as $E_{s}^{f}$ and $E_{s}^{o}$ respectively. The extraction of the sulcal edges associated to an interrupted sulcus remains identical. The sulcus will be described as two connected graphs through the minimum spanning tree. However, the interruptions will appear as holes in the distribution of points along the respective path. This procedure yields a set of folding features distributed across the entire cortical surface as 


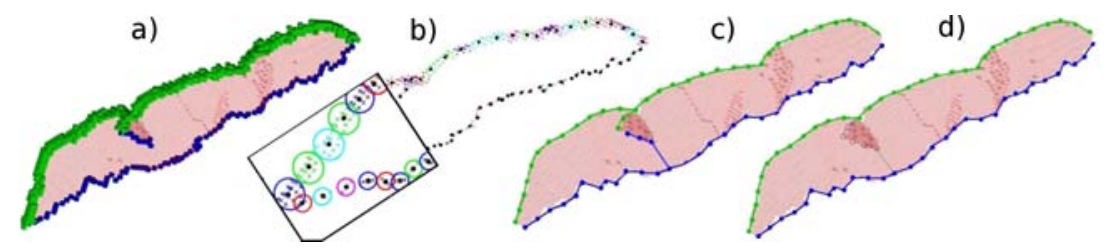

Fig. 2. a) Each sulcus is decomposed into three subsets of voxels: fundus (in blue), outer edge (green) and other voxels (in red) using brainVISA. The description of each sulcus may be summarized by fundus and outer border voxel subsets. b) Those voxels are grouped into clusters (shown here as colored circles) and each cluster is reduced to its barycenter (black dots). Finally, the sulcal borders illustrated in c) are reduced to simple lines in d) through a longest path approach.

illustrated Fig[1 c). These features hereby define the sulcal imprint of each individual brain anatomy $-\mathcal{I}=\left[E_{1}^{f}, E_{1}^{o}, . ., E_{s}^{f}, E_{s}^{o}, . ., E_{S}^{f}, E_{S}^{o}\right]$ - that will be matched across subjects.

\section{Measure-Based Diffeomorphic Matching of Sulcal Imprints}

We introduce a non-linear pairwise registration approach of sulcal imprints in the general framework of the LDDMM theory [5]. The deformation $\phi$ of sulcal edge $E_{1}$ onto another sulcal edge $E_{2}$ is defined as the minimum of the following registration energy functional:

$$
J_{E_{1}, E_{2}}^{\text {sulc }}(\phi)=\gamma \operatorname{Reg}(\phi)+\operatorname{Mis}\left(\phi\left(E_{1}\right), E_{2}\right),
$$

where the first term controls for the regularity of the deformation while the second term evaluates the mismatch between the deformed sulcal edge $\phi\left(E_{1}\right)$ and the target $E_{2} ; \gamma$ being a scalar trade-off parameter. Following the LDDMM theory, $\phi$ is a diffeomorphism if it may be defined as a solution at time $t=1$ of the differential equation: $\partial_{t} \phi_{t}^{\mathbf{v}}=\mathbf{v}_{t} \circ \phi_{t}^{\mathbf{v}}$, with initial condition $\phi_{0}^{\mathbf{v}}=I d$. Id represents the identity deformation that maps an object onto itself. In this equation, $\mathbf{v}_{t}: \mathbb{R}^{3} \longrightarrow \mathbb{R}^{3}$ is a time-dependent vector field which models the infinitesimal variations of the deformation flow. $\mathbf{v}_{t}$ belongs to the reproducing kernel Hilbert space $V$ of regular vector fields. $V$ is associated to a kernel $K^{V}$ controlling for the regularity of the final diffeomorphic transform. We define the cost of a given diffeomorphism $\phi$ as its distance to the identity transform: $d_{V}^{2}(I d, \phi)=\inf _{\mathbf{v}}\left\{\int_{0}^{1}\left\|\mathbf{v}_{t}\right\|_{V}^{2} d t, \phi_{1}^{\mathbf{v}}=\phi\right\}$. Registering a pair of sulcal edges in a diffeomorphic framework then consists in minimizing the functional:

$$
J_{E_{1}, E_{2}}^{\text {sulc }}(\phi)=\gamma d_{V}^{2}(i d, \phi)+\operatorname{Mis}\left(\phi\left(E_{1}\right), E_{2}\right) .
$$

The mismatch Mis between two sulcal edges is defined hereafter. 


\subsection{Description of Sulcal Landmarks as Measures}

Each couple of anatomically-corresponding sulcal edges are considered as two sets of points $E_{1}=\left(x_{i}\right)_{i<n_{x}}$ and $E_{2}=\left(y_{j}\right)_{j<n_{y}} \subset \mathbb{R}^{3}$, with possibly $n_{x} \neq n_{y}$. These two sets of points can be described mathematically as measures $\mu$ and $\nu$ respectively, each consisting of the weighted sum of Dirac distributions [5: $\mu=\sum_{i=1}^{n_{x}} a_{i} \delta_{x_{i}}$ and $\nu=\sum_{i=1}^{n_{y}} b_{i} \delta_{y_{i}} .\left(a_{i}\right)_{i<n_{x}}$ and $\left(b_{j}\right)_{j<n_{y}}$ are two sets of scalar weight parameters. These weights are set as follows: if the neighborhood of each $x_{i}$ in the associated sulcal edge is denoted as $H$, then $a_{i}=$ $\frac{1}{\operatorname{card}(H)} \sum_{h \mid h \in H}\left\|x_{h}-x_{i}\right\|_{\mathbb{R}^{3}}$. This uniformly distributes the weights along the measure, thereby compensating for the heterogeneity in the spatial distribution of points along the edge lines corresponding to interrupted sulci. The action of $\phi$ on the measure $\mu$ may be defined as a mass transportation problem: $\phi(\mu)=$ $\phi\left(\sum_{i} a_{i} \delta_{x_{i}}\right)=\sum_{i} a_{i} \delta_{\phi\left(x_{i}\right)}$. In order to evaluate the adjustment of the source sulcal measure $\mu$ to the target measure $\nu$, we introduce an additional reproducing kernel Hilbert space $I$ associated to a second kernel $K^{I}$, such that every bounded and signed measure belongs to $I^{*}$, the dual space of $I$. We may then evaluate the distance between the pair of measures $\mu$ and $\nu$ as: $d_{I}^{2}(\phi(\mu), \nu)=\|\phi(\mu)-\nu\|_{I^{*}}^{2}=$ $\sum_{i, j} a_{i} a_{j} K^{I}\left(\phi\left(x_{i}\right), \phi\left(x_{j}\right)\right)+\sum_{i, j} b_{i} b_{j} K^{I}\left(y_{i}, y_{j}\right)-2 \sum_{i, j} a_{i} b_{j} K^{I}\left(\phi\left(x_{i}\right), y_{j}\right)$. Equation (2) can therefore be rewritten as:

$$
J_{\mu, \nu}^{\text {sulc }}(\phi)=\gamma d_{V}^{2}(i d, \phi)+\|\phi(\mu)-\nu\|_{I^{*}}^{2} .
$$

Considering now two sulcal imprints with $P$ sulcal edges in common, we define $\mathcal{I}_{1}=\left[\mu^{1}, . ., \mu^{P}\right]$, the source sulcal imprint to be adjusted to a target sulcal imprint $\mathcal{I}_{2}=\left[\nu^{1}, . ., \nu^{P}\right]$. Registering a pair of brains through their respective sulcal imprints corresponds to the minimization of the following functional:

$$
J_{\mathcal{I}_{1}, \mathcal{I}_{2}}^{i m p r}(\phi)=\gamma d_{V}^{2}(i d, \phi)+\sum_{p=1}^{P}\left\|\phi\left(\mu^{p}\right)-\nu^{p}\right\|_{I^{*}}^{2} .
$$

Of primary importance is that the resulting deformation is a fully $3 \mathrm{D}$ diffeomorphic map defined everywhere in $\mathbb{R}^{3}$, hence not only on the cortical surface, but also in the entire MRI volume.

\subsection{Unbiased, Empirical Anatomical Template for Multiple-Subject Registration}

We now propose a multi-scale iterative approach at the group level that extends previous results from [6]. This approach avoids the arbitrary selection of a single brain as a registration template while exploiting the maximum of the sulcal information available. The methodology involved is itemized as follows:

1. The sulcal imprint is extracted from each individual brain data and is linearly registered into standardized Talairach space $[3$.

2. An empirical template is defined as the union of the entire set of sulcal points through the entire group of subjects involved in the study. For each sulcal 
label available across the group, the corresponding sulcal landmark in the template consists of the union of all points associated to this label within every subject of the group.

3. Diffeomorphic transformation of each individual data onto the empirical template is operated following the methodology described in Section 3

4. The process in steps 2 and 3 is iterated $Q$ times by considering the resulting transformed sulcal points as the new running template, until the evolution between two successive template samples is inferior to a fixed threshold. We focus on finer scale deformations as the template is iteratively refined by reducing the sizes of the kernels involved in the registration energy (for both regularization and mismatch terms in Eq. (5) between two iterations.

Therefore, registering $N$ subjects consists of $Q$ iterations of $N$ minimization steps while the empirical template is updated between two iterations, in a multi-scale framework.

For clarity purposes, we now detail the elaboration of an empirical template consisting of a single sulcus through a group of $N$ individual sulcal imprints. The generalization to $P$ sulcal labels extends the strategy introduced by Eq. (2) through Eq. (4). Following [6], let us denote $\left(x_{i p}\right)_{1 \leq i \leq N, 1 \leq p \leq n_{i}}$, the $N$ individual sets consisting of $n_{i}$ points describing the sulcus to be matched across subjects; $a_{i p} \in \mathbb{R}$ their associated weights and $\mu_{i}=\sum_{p=1}^{n_{i}} a_{i p} \delta_{x_{i} p}$, their respective measure form. Obtaining the measure $\mu$ of the group template may be defined as a minimisation problem: $\left\{\hat{\phi}_{i}, \hat{\mu}\right\}=\arg \min _{\phi_{i}, \mu} \sum_{i=1}^{N}\left\{\gamma d_{V}^{2}\left(i d, \phi_{i}\right)+\left\|\phi_{i}\left(\mu_{i}\right)-\mu\right\|_{I^{*}}^{2}\right\}$. Note that for fixed $\phi_{i}, \hat{\mu}$ reduces to the sum of the Dirac masses associated to the union of all points $\phi_{i}\left(x_{i p}\right): \hat{\mu}=\frac{1}{N} \sum_{i=1}^{N} \phi_{i}\left(\mu_{i}\right)=\frac{1}{N} \sum_{i=1}^{N} \sum_{p=1}^{n_{i}} a_{i p} \delta_{\phi_{i}\left(x_{i p}\right)}$. Hence, the problem reduces to:

$$
\left\{\hat{\phi}_{i}\right\}=\arg \min _{\phi_{i}} \sum_{i=1}^{N}\left\{\gamma d_{V}^{2}\left(i d, \phi_{i}\right)+\left\|\phi_{i}\left(\mu_{i}\right)-\frac{1}{N} \sum_{i=1}^{N} \phi_{i}\left(\mu_{i}\right)\right\|_{I^{*}}^{2}\right\} .
$$

At the end of the process, the transformation that brings subject $i$ into the common space is the composition of $Q$ diffeomorphisms: $\phi_{i}^{Q} \circ \phi_{i}^{Q-1} \circ \ldots \circ \phi_{i}^{1}$. By definition, the composition of $Q$ such diffeomorphisms is also diffeomorphic.

\section{Results}

DISCO was applied for evaluation purposes to the registration of the brains from 20 healthy subjects through the elaboration of their corresponding empirical anatomical template. Sulcus automatic labeling was checked for errors manually. Between 88 and 98 sulci were identified across subjects in this group, 71 of which were common to all subjects and only 8 sulci were found in less than 14 of the subjects. The iterative sulcal template refinement process converged though $Q=9$ iterations with template update. Computation time on a cluster of 10 processors running at $3.6 \mathrm{GHz}$ with $2 \mathrm{~Gb}$ of RAM was 10 hours.

As shown on Fig 3 , the superimposition of most sulcal edges is clearly improved after diffeomorphic transformation using DISCO. Further, it is important 

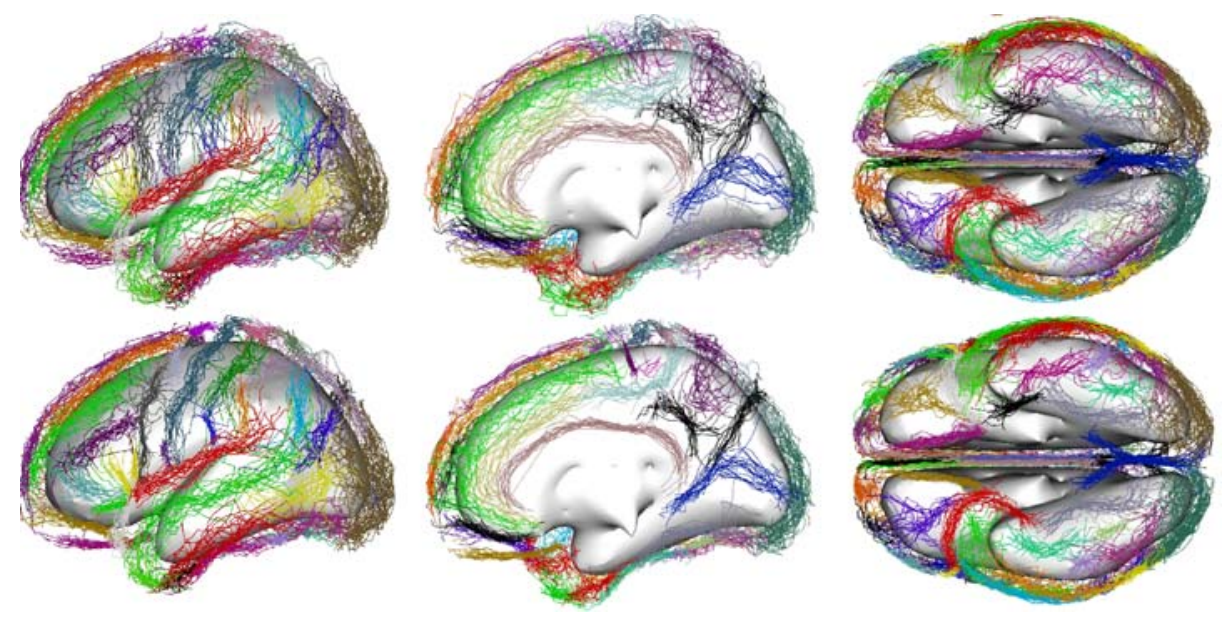

Fig. 3. Comparative results of the registration of 20 brains using the linear technique 3] (upper panel) vs. DISCO diffeomorphic sulcal matching (lower panel)
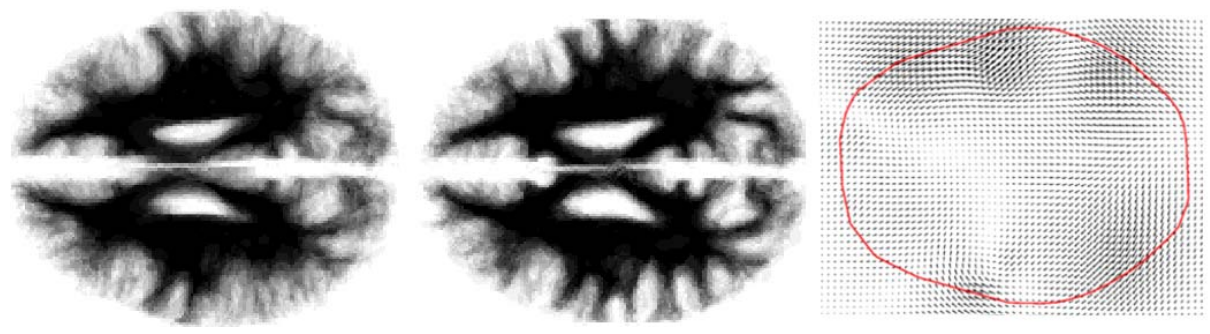

Fig. 4. Comparison of the linear (left) and DISCO (middle) registration procedures across 20 white-matter masks. Correct alignment of cortical circumvolutions would yield reduced fuzziness in the gray levels of the average volume masks, as readily observed after DISCO was applied. Right: a typical slice of the resulting DISCO deformation field. The convex hull of the brain in that particular slice is shown in red.

to remember that matching is not warping, as some aspects of the original group variability have been preserved by the regularity of the diffeomorphic transforms.

The Hausdorff distance between each subject and the rest of the group was computed for each sulcal landmark, and averaged across subjects, after linear or diffeomorphic matching. This measure is an indicator of the spatial dispersion of sulcal edges. The Hausdorff distance was reduced by $18 \%$ (from $17.2 \mathrm{~mm}$ to $14 \mathrm{~mm}$ ) on average across all sulci and subjects using the diffeomorphic matching compared to the linear registration approach. For instance, the sulcal dispersion has been decreased by more than $7 \mathrm{~mm}$ in the inferior part of the temporal lobe. The improvement was smaller in regions with sulcal objects of greater geometrical irregularity across subjects as in e.g. the occipital lobe. The dispersion of the central sulcus was reduced by "only" $1.6 \mathrm{~mm}$ as it had been already correctly realigned by the 
linear registration procedure. Note also that the deformation of any given sulcus is tempered by the transformations occurring in its neighborhood.

As discussed in Section 3 , the DISCO transformation naturally extends to the entire 3D volume as illustrated Fig 4 through the group averages of the binary white-matter masks.

\section{Conclusion}

The suggested approach combines the attractive properties of diffeomorphic matching with the pairing of anatomical landmarks considered by neuroanatomical experts. Future work will focus on larger-scale validation, including comparison with other non-linear registration methods. The preliminary results presented here suggest that this technique may lead to a new systematic approach for anatomical registration in neuroimaging group studies.

\section{References}

1. BrainVISA/Anatomist website, http://brainvisa.info

2. Ashburner, J.: A fast diffeomorphic image registration algorithm. Neuroimage 38, 95-113 (2007)

3. Collins, D.L., Neelin, P., Peters, T.M., Evans, A.C.: Automatic 3D Intersubject Registration of MR Volumetric Data in Standardized Talairach Space. J. Comp. Assist. Tomog. 18, 192-205 (1994)

4. Fischl, B., Rajendran, N., Busa, E., Augustinack, J., Hinds, O., Yeo, B.T., Mohlberg, H., Amunts, K., Zilles, K.: Cortical Folding Patterns and Predicting Cytoarchitecture. Cerebral Cortex (2007)

5. Glaunès, J., Trouvé, A., Younes, L.: Diffeomorphic matching of distributions: a new approach for unlabelled point-sets and sub-manifolds matching. In: Proc. IEEE Conf. Comp. Vis. Pat. Rec., vol. 2, pp. 712-718 (2004)

6. Glaunès, J., Joshi, S.: Template estimation form unlabeled point set data and surfaces for computational anatomy. In: MICCAI 2006. LNCS, pp. 58-65. Springer, Heidelberg (2006)

7. Hellier, P., Barillot, C.: Cooperation between local and global approaches to register brain images. In: Insana, M.F., Leahy, R.M. (eds.) IPMI 2001. LNCS, vol. 2082, pp. 315-328. Springer, Heidelberg (2001)

8. Joshi, A.A., Shattuck, D.W., Thompson, P.M., Leahy, R.M.: Surface-Constrained Volumetric Brain Registration Using Harmonic Mappings. IEEE Trans. Med. Imag. 26, 1657-1669 (2007)

9. Liu, T., Shen, D., Davatzikos, C.: Deformable registration of cortical structures via hybrid volumetric and surface warping. Neuroimage 22, 1790-1801 (2004)

10. Mangin, J.-F., Rivière, D., Cachia, A., Duchesnay, E., Cointepas, Y., Papadopoulos-Orfanos, D., Scifo, P., Ochiai, T., Brunelle, F., Régis, J.: A framework to study the cortical folding patterns. Neuroimage 23, 129-138 (2004)

11. Perrot, M., Rivière, D., Tucholka, A., Mangin, J.-F.: Joint Bayesian Cortical Sulci Recognition and Spatial Normalization. In: IPMI. LNCS. Springer, Heidelberg (to appear) 
12. Postelnicu, G.M., Zöllei, L., Fischl, B.: Combined Volumetric and Surface Registration. IEEE Trans. Med. Imag. 28, 508-522 (2008)

13. Qiu, A., Miller, M.I.: Cortical hemisphere registration via large deformation diffeomorphic metric curve mapping. In: Ayache, N., Ourselin, S., Maeder, A. (eds.) MICCAI 2007, Part I. LNCS, vol. 4791, pp. 186-193. Springer, Heidelberg (2007)

14. Shi, Y., Thompson, P.M., Dinov, I., Osher, S., Toga, A.W.: Direct cortical mapping via solving partial differential equations on implicit surfaces. Med. Imag. Anal. 11, 207-223 (2007) 